\title{
Mitarbeiterinfektionsschutz in Zeiten der Coronavirus-Pandemie: Die akute Implementierung von telemedizinischen Mitarbeiterkonferenzen „von Null auf Hundert“
}

\author{
Protecting Medical Professionals from Infection During the \\ Coronavirus Pandemic: Overnight Implementation of Virtual \\ Medical Boards
}

\author{
Autoren \\ Tatjana Tamara König® ${ }^{\mathbb{D}}$, Anna-Franziska Lenz, Jan Goedeke, Oliver J. Muensterer
}

Institut

Klinik und Poliklinik für Kinderchirurgie, Universitätsmedizin Mainz, Mainz

Schlüsselwörter

Coronavirus, Telemedizin, Datenschutz, Virtuelles Board

Key words

Corona Virus, Telemedicine, Virtual Board, Patient Privacy

Bibliografie

DOI https://doi.org/10.1055/a-1165-1878

Published online: 15.5 .2020

Gesundheitswesen 2020; 82: 497-500

(c) Georg Thieme Verlag KG Stuttgart · New York

ISSN 0941-3790

\section{Korrespondenzadresse}

Dr. Tatjana Tamara König

Klinik und Poliklinik für Kinderchirurgie

Universitätsmedizin Mainz

Langenbeckstraße 1

55131 Mainz

tatjana.koenig@unimedizin-mainz.de

\section{ZUSAMMENFASSUNG}

Ziel der Studie Die geltenden verschärften Hygieneempfehlungen des Robert-Koch-Instituts zur Eindämmung der Ausbreitung des Coronavirus mit Versammlungsverbot und Mindestabstand sind im innerklinischen Alltag nicht umsetzbar. Zur Minimierung von persönlichen Kontakten wurden die internen Arztkonferenzen auf ein Videokonferenzformat umgestellt. Methodik Innerhalb 24 Stunden wurde unter Anschaffung von Standard-Webcams, Auswahl eines lizenzierten Videoanbieters und Ausnutzen der bereits vorhandenen technischen Geräte die allgemeine Hygienerichtlinie für unsere Mitarbeiter umgesetzt. Häufige Fehlerquellen im Rahmen der Videokonferenz wurden protokolliert und technische Lösungen erarbeitet.
Ergebnisse Die Videokonferenz ermöglichte die adäquate Patientenversorgung und Aufrechterhaltung der gewohnten organisatorischen Abläufe. In 4 Wochen konnten 956 persönliche Kontakte vermieden werden. Die meisten Fehler traten im Rahmen der ersten Teilnahme einer Person oder Erstanmeldung eines Gerätes auf. Die häufigsten Fehler betrafen die Tonqualität und konnten umgangen werden, indem nur an der Diskussion beteiligte Teilnehmer ihr Mikrofon für die Sprechdauer aktivierten.

Schlussfolgerung Mitarbeiterkonferenzen im Videoformat können mit geringem personellem und technischem Aufwand etabliert werden, um der Ausbreitung des Coronavirus unter medizinischem Personal entgegenzuwirken.

\footnotetext{
ABSTRACT

Background For medical professionals in hospitals, it is nearly impossible to abide by the rules of social distancing to prevent coronavirus transmission. In order to avoid unnecessary contact between medical staff, virtual medical boards were implemented.

Methods Technical requirements for virtual boards were established overnight. Standard web cams and one license for a licensed provider of virtual boards were acquired. Most of the other technical gear required was readily available. Frequent and typical errors during the virtual boards were recorded and analyzed.

Results Virtual boards provided the means for adequate patient care in spite of social distancing among the medical staff. Over a period of 4 weeks, 956 face-to-face contacts were avoided. Most errors occurred during first-time participation and concerned audio transmission. By muting the microphone of inactive participants, most of these errors could be avoided.

Conclusions Virtual boards for medical staff can be implemented with minimal effort und standard technical gear to slow down the transmission of coronavirus among medical professionals in the hospital setting.
} 


\section{Einleitung}

Die aktuelle Coronavirus-Pandemie stellt neben der medizinischen Patientenversorgung auch in Bezug auf die Kommunikation von Arzt zu Patient, aber auch Arzt zu Arzt eine große Herausforderung dar. Die Empfehlungen des Robert-Koch-Instituts (RKI) zum „Social Distancing " sind im Rahmen der täglichen klinischen Arbeit im Krankenhaus in der bisherigen Arbeitsweise nicht umzusetzen. Neue Herangehensweisen sind dringend erforderlich, um die Anzahl der Kontakte soweit wie möglich zu minimieren. Unter diesen Umständen hat die Kinderchirurgie der Universitätsmedizin Mainz ab dem 16.03.2020 innerhalb eines Tages die konsequente Durchführung aller Mitarbeiterkonferenzen per Videokonferenzsystem umgesetzt.

Das System hat sich rasch in unserem klinischen Alltag etabliert. Um anderen Kliniken die Umsetzung von telemedizinischen Konferenzlösungen zu erleichtern, beschreiben wir hier die mit einer solchen Umstellung einhergehenden Herausforderungen, Fehlerquellen, aber auch die Chancen und Möglichkeiten in einem initialen Erfahrungsbericht.

\section{Material und Methoden}

\section{Durchführung}

Seit dem 16.03.2020 wurden die morgendliche Frühbesprechung, Oberarztbesprechung, wissenschaftliche Arbeitsgruppentreffen und die Fortbildungsveranstaltungen unsere Abteilung auf ein telemedizinisches Format umgestellt. Zur Durchführung der Konferenzen wurde eine von der Kassenärztlichen Bundesvereinigung (KBV) zertifizierte, web-basierte Software genutzt. Die Web-Konferenz wurde am Vortag vom Initiator angelegt. Die PrivatsphäreEinstellungen des Videoanbieters wurden so gewählt, dass sich keine externen User unter Benutzung des Web-Links in die Konferenz einwählen können. Die eingeladenen Teilnehmer erhielten einen E-Mail-Link zur Teilnahme an der Konferenz, welche durch den Initiator eröffnet wurde. Um beizutreten, wurde der Link aus dem E-Mail-Postfach mit Google Chrome oder Apple Safari geöffnet. Vorab wurde von allen Usern an dem jeweiligen Endgerät der Gerätecheck der Software von MEDITyme durchgeführt, um die Einsatzfähigkeit der Hardware und Internetverbindung zu überprüfen. Die Software ermöglicht eine Videokonferenz mit separater Chat- (Versenden von simultanen Textnachrichten), sowie eine Screenshare-Funktion, bei der jeder Teilnehmer seinen Bildschirm zur gemeinsamen Begutachtung von Befunden wie Röntgenbildern, Laborwerten oder anderen Dokumenten freigeben kann. Darüber hinaus können Dateien mit der Gruppe geteilt und lokal geöffnet werden. Die inhaltliche Struktur, der Zeitpunkt und der zeitliche Umfang der Veranstaltungen wurde unverändert zum präpandemischen Vorgehen beibehalten.

\section{Technische Voraussetzungen}

Die Besprechungen wurden hauptsächelich an den Standardarbeitsplätzen der Ärzte in der Klinik, teilweise aber auch von zu Hause oder mobil über eine LTE-Verbindung auf Smartphones durchgeführt. Eine besondere Rechnerleistung, Konfiguration oder ein spezielles Mobiltelefon waren nicht erforderlich. Jeder Arbeitsplatz in der Klinik wurde mit einer Standard-HD-Webcam (Video- anrufe im Breitbildformat in 720p, USB 2.0 Anschluss, Sichtfeld: $60^{\circ}$ ) ausgestattet. Um die Einhaltung des empfohlenen Mindestabstandes von 1,5 m zu gewährleisten, war es darüber hinaus möglich, sich an Arbeitsplätzen ohne Webcam einzuwählen und ein Headset (z. B. Headset des Mobiltelefons) zur Kommunikation zu nutzen. Zwei bis drei Teilnehmer nahmen mit Laptops mit integrierter Webcam aus dem Home-Office teil. Da Smartphones standardmäßig mit Kamera und Mikrofon ausgestattet, ermöglichten sie eine Teilnahme an Videokonferenzen ohne Hardwareergänzungen. Um Nebengeräusche zu minimieren, wurden alle Teilnehmer, die nicht aktiv an der Diskussion beteiligt waren, angehalten, ihre Mikrofone während der inaktiven Zeit zu deaktivieren.

\section{Software}

Bei der hier verwendeten telemedizinischen Software (MEDITyme $\mathrm{GmbH}$, Starnberg, Deutschland) handelt es sich um eine web-basierte Applikation. Die Installation von zusätzlicher Software ist nicht erforderlich. Um die Funktionen des Videodienstes optimal nutzen zu können, wurden nach den Empfehlungen der Firma MEDITyme die aktuelle Version von Google Chrome oder Apple Safari als Webbrowser verwendet.

\section{Evaluation}

Die Konferenzen wurden prospektiv anhand eines standardisierten Protokolls bewertet. Aufgetretene Fehler wurden protokolliert und in Kategorien eingeteilt: Software, Hardware, Internetverbindung, Bedienungsfehler.

\section{Ergebnisse}

Zwischen dem 16.03. und 09.04.2020 wurden 18 Frühbesprechungen, 5 wissenschaftliche Arbeitsgruppentreffen, 5 interne Fortbildungsveranstaltungen und 2 Oberarztkonferenzen durchgeführt. Teilgenommen haben 11 ÄrztInnen, 2 Studierende, 4 wissenschaftliche Mitarbeiterlnnen und MitarbeiterInnen aus Sekretariat und Pflege. Der Altersdurchschnitt der Teilnehmer betrug 39,2 \pm 9,3 Jahre. Maximal 13 Teilnehmer nahmen gleichzeitig an den Konferenzen teil, die durchschnittliche Teilnehmerzahl belief sich auf 8 Personen. Über einen Zeitraum von 4 Wochen konnten so insgesamt 956 persönliche Kontakte vermieden werden. Bild- und Tonqualität mit der beschriebenen Hardwareausstattung wurden von allen Teilnehmern als ausreichend zur adäquaten Durchführung der Konferenzen bewertet.

Von 30 durchgeführten Konferenzen musste eine aufgrund technischer Probleme abgesagt werden. In diesem Fall führte ein hoher Datentraffic durch ein automatisches Update des Computers der Initiatorin zu einem wiederholten Abstürzen der Konferenzschaltung. Die meisten Fehler traten auf, wenn Teilnehmer zum ersten Mal an einer Konferenz teilnahmen. Häufige technische Probleme, die nicht zum Abbruch einer Konferenz führten, betrafen insbesondere die Tonübertragung in Form von akustischen Rückkopplungen. Diese entstanden vor allem dadurch, dass mehrere Konferenzteilnehmer von verschiedenen Geräten im gleichen Raum ohne Headsets an der Konferenz teilnahmen. Ist die Datenleitung der Teilnehmer unterschiedlich schnell, kann es außerdem zu unangenehmen Echoeffekten kommen, bei denen sich Teilnehmer selbst zeitversetzt über die Mikrofone der anderen Teilnehmer 
hören. Beide Probleme konnte durch die Nutzung von Headsets oder einfache Inaktivierung der Mikrofone der passiven Teilnehmer verhindert werden.

Andere Fehler ergaben sich durch die Nutzung veralteter Versionen des Webbrowsers oder insgesamt fehlende Kompatibilität mit dem verwendeten Webbrowser. Im Browser muss vorab darauf geachtet werden, dass Kamera und Mikrofon jeweils separat zur Nutzung freigegeben sind. Zusätzlich können individuelle Sicherheits- und Datenschutzeinstellungen der Arbeitsplätze so konfiguriert sein, dass die Nutzung von Kamera und/ oder Mikrofon in der Grundeinstellung blockiert sind. In diesen Fällen ist eine passive Teilnahme als Zuschauer und -hörer möglich, zur aktiven Konferenzteilnahme muss eine Adaptation der Einstellungen am jeweiligen Computer durch den Systemadministrator erfolgen. Im Rahmen der Besprechungen wurden Röntgen-CT, MRT-Bilder, Texte und Präsentationsfolien auf dem Bildschirm geteilt. Hatte der präsentierende Arzt nur einen Bildschirm zur Verfügung, kam es in einigen Fällen zu optischen Rückkopplungen. Diese Art von Rückkopplung konnten vermieden werden, indem die Präsentationen von Arbeitsplätzen erfolgten, die mit 2 Monitoren ausgestattet waren.

Bei verringerter Datenübertragungsgeschwindigkeit wurden die Teilnehmer angehalten, ihre Kameras zu inaktivieren. Dadurch konnte in allen Fällen eine gute auditive Teilnahme an der Videokonferenz erreicht werden. Bei deaktivierter Kamera am eigenen Arbeitsplatz sind die anderen Teilnehmer und geteilten Inhalte weiterhin sichtbar. Eine kabelgebundene LAN-Verbindung per Ethernet-Kabel war der WLAN-Verbindungen in einem Fall qualitativ überlegen und führte zu einer beschleunigten Datenübertragungsrate mit besserer Übertragungsqualität.

Mobile Endgeräte eigneten sich aufgrund der kleineren Bildschirmgröße und fehlenden Einbindung in das Krankenhausinformationssystem nicht zur Moderation der Konferenz, ermöglichten jedoch eine problemlose Teilnahme mit guter Bild- und Tonqualität über das LTE-Netz.

\section{Diskussion}

Die Coronavirus-Pandemie hat unser Gesundheitswesen in kürzester Zeit vor kaum vorstellbare Herausforderungen gestellt. Eine gute medizinische Versorgung hängt von einer engen Kommunikation zwischen Ärzten, angrenzende Berufsgruppen und den Patienten ab. Gleichzeitig ist es unbedingt erforderlich, den körperlichen Abstand zu wahren, um die Ausbreitung des SARS-CoV-2 Virus zu verlangsamen. Um die medizinische Versorgung in der Krise sicherzustellen, kommt dem Infektionsschutz beim medizinischen Personal eine besondere Bedeutung zu. So haben wir an unserer Klinik frühzeitig die Entscheidung getroffen, im Hinblick auf die Minimierung des körperlichen Kontaktes unter den Mitarbeitern, alle ärztlichen Konferenzen an unserer Klinik in ein Videoformat zu überführen.

Die ausgewählte telemedizinische Lösung konnte innerhalb eines Tages in den täglichen Ablauf implementiert werden und wurde von allen Mitarbeitern unproblematisch umgesetzt. Der größte Teil der notwendigen Infrastruktur war im Vorfeld bereits vorhanden. Es war lediglich die Anschaffung des Zugangs des Videokonferenzanbieters und 5 zusätzlicher Standard-Webcams aus
Klinikmitteln erforderlich. Durch die web-basierte Applikation waren weder die Installation zusätzlicher Software durch den Systemadministrator, noch eine Anpassung der Klinik-Firewall erforderlich. Bild- und Tonqualität waren ausreichend, um eine adäquate medizinische Versorgung der Patienten sicherzustellen.

Vorhandene internetfähige Geräte wie Smartphones oder Tablets konnten ebenfalls problemlos im LTE-Netz genutzt werden. Hier war der Funktionsumfang durch die kleinere Displaygröße und fehlenden Zugriff auf das Krankenhausinformationssystem eingeschränkt. Daher war mit diesen Geräten die Teilnahme, aber nicht eine Präsentation im Rahmen einer Konferenz möglich. Dennoch konnte durch den Einsatz mobiler Endgeräte eine weitere räumliche Entzerrung und Einhaltung des empfohlenen Mindestabstands zwischen den Teilnehmern erreicht werden. Bei der Nutzung von privaten mobilen Endgeräten sollte aus Datenschutzgründen kein Download von personenbezogenen Daten erfolgen.

Die Videokonferenzen ermöglichten so eine adäquate Versorgung der Patienten bei gleichzeitiger Minimierung des physischen Kontakts der Mitarbeiter. Auch die Teilnahme aus dem Homeoffice war problemlos möglich. Die Tagesstruktur und grundlegende Organisation der Abteilung, sowie die interkollegiale fachliche Kommunikation konnten beibehalten werden, ohne die derzeitig erforderlichen Hygieneempfehlungen zu kompromittieren. Natürlich sind diese Maßnahmen nur dann sinnvoll, wenn die Mitarbeiter sich auch außerhalb der Konferenzen an die Hygienevorschriften und Abstandsregelung halten.

Insbesondere bei steigender Teilnehmerzahl ist ein hohes Maß an Konzentration und Disziplin der Teilnehmer erforderlich. Gleichzeitiges oder lautes Sprechen, v. a. bei Verzögerungen in der Datenleitung einiger Teilnehmer, erschwert die Diskussion erheblich. Die meisten akustischen Störungen können jedoch dadurch vermieden werden, dass jeder inaktive Teilnehmer sein Mikrofon konsequent inaktiviert.

Gravierende technische Probleme, die zu einem Abbruch der Konferenz führten, traten nur in einem Fall aufgrund eines erhöhten Datentraffics auf dem Computer der Initiatorin auf. Weitere Fehler bezogen sich v. a. auf die Tonqualität. Vermeidbare Fehlerquellen sollten im Vorfeld einer Konferenz von jedem Teilnehmer ausgeschlossen werden ( Tab. 1: Do's and Don'ts).

Zu den Nachteilen gehört, dass trotz guter Beurteilbarkeit die offiziell geforderte Mindestauflösung zur Befundung von Röntgenbildern durch Teilen des Bildschirms in Abhängigkeit des anzeigenden Gerätes nicht erreicht werden kann. In diesem Fall muss jeder Teilnehmer das Bild separat auf seinem eigenen Computer öffnen.

Für die ambulante Patientenversorgung mittels Telemedizin gibt es klare Vorgaben der Kassenärztlichen Bundesvereinigung (KBV). Die Abrechnung einer Videosprechstunde nach §291g SGB V ist in der Anlage 31b zum Bundesmantelvertrag Ärzte (BMV-Ä) geregelt [1]. Im Eilverfahren wurden im Rahmen der aktuellen Corona Lage die Beschränkungen für die Abrechnung der Videosprechstunde ab dem 01.04.2020 durch die KBV gelockert und für alle Indikationen geöffnet. Zum Beispiel ist es in Zukunft möglich, mehr als $20 \%$ der Sprechstunde telemedizinisch durchzuführen und auch abzurechnen. Die Voraussetzungen für den Datenschutz bleiben jedoch unverändert: neben der Einwilligung des Patienten und geeigneten räumlichen Voraussetzungen fordert die KBV eine End-zu-EndVerschlüsselung der Videokonferenz. Voraussetzung für die Ab- 
- Tab. 1 Do's and Don'ts: Vermeidbare Fehler bei der Durchführung von Videokonferenzen.

\begin{tabular}{|l|}
\hline Do's \\
\hline $\begin{array}{l}\text { Sicherstellung eines mit der Software kompatiblen Browsers/ Browser- } \\
\text { version }\end{array}$ \\
\hline $\begin{array}{l}\text { Freigabe von Zugriff des entsprechenden Browsers auf Kamera und } \\
\text { Mikrofon des Endgerätes }\end{array}$ \\
\hline Synchronisierung und automatische Update-Funktion deaktivieren \\
\hline Testen der Hardware und Internetverbindung vor Beginn der Konferenz \\
\hline $\begin{array}{l}\text { Verwendung eines Arbeitsplatzes mit 2 Monitoren durch den Präsentie- } \\
\text { renden }\end{array}$ \\
\hline $\begin{array}{l}\text { Nutzung eines Headsets bei bekanntermaßen eingeschränkter } \\
\text { Datenübertragungsrate }\end{array}$ \\
\hline $\begin{array}{l}\text { Nutzung eines Verbindungskabels anstelle von W-LAN bei bekannterma- } \\
\text { ßen eingeschränkter Datenübertragungsrate }\end{array}$ \\
\hline Don'ts \\
\hline synchrone Nutzung anderer Anwendungen mit Zugriff auf Kamera-, \\
Mikrofon- oder Lautsprecher \\
\hline $\begin{array}{l}\text { Einstecken oder Entfernen von Hardware (z. B. Headsets) während einer } \\
\text { laufenden Konferenz }\end{array}$ \\
\hline Gleichzeitiger Up-/ oder Downloaden großer Datenmengen \\
\hline Teilen des Bildschirmes, auf dem die Konferenz aktiv ist \\
\hline Teilnahme mehrerer Personen ohne Headset in einem Raum \\
\hline Download personenbezogener Daten auf private Geräte \\
\hline
\end{tabular}

rechnung ist die Nutzung eines der KBV-zertifizierten Videodienstanbieter [2]. Technisch unterscheiden sich die Anbieter unter anderem dadurch, dass einige rein web-basiert betrieben werden und andere die Installation zusätzlicher Software erfordern, die im klinischen Umfeld durch den Nutzer selbst meist unmöglich ist.

I Bezug auf Datenschutz und technische Vorgaben gibt es für die innerklinische Nutzung von telemedizinischer Software außerhalb der Teleradiologie aktuell keine eindeutigen Vorgaben. Die innerklinische Kommunikation fällt nicht in den Zuständigkeitsbereich der KBV. Aus Sicht der Bundesärztekammer ist die Zulässigkeit der Arzt-zu-Arzt-Videokonferenz in den „Hinweisen und Erläuterungen zum Fernbehandlungsverbot nach § 7 Absatz 4 MBO-Ä“ [3] beschrieben, technische und datenschutzrechtliche Grundlagen wurden hier jedoch nicht definiert. Die technischen Anforderungen für die eine ärztliche Konferenz (z. B. im Rahmen eines Tumorboards) gehen deutlich über die Anforderungen einer 1:1-Videosprechstunde zwischen Arzt und Patient hinaus: sie erfordern das Zusammenschalten von mehreren Personen und ein Mindestmaß an Bildqualität zur gemeinsamen Befundbeurteilung. Aktuell kann keiner der KBV-zertifizierten Videodienstanbieter in Deutschland eine End-zu-End Verschlüsselung für Videokonferenzen mit mehr als 2 Parteien gewährleisten. Datenschutzrechtlich befinden wir uns in einer Grauzone.

Zusammenfassend eröffnet die telemedizinische Arztkonferenz die Möglichkeit, bewährte Abläufe im klinischen Alltag beizubehalten und zugleich Versammlungen mehrerer Menschen mit Unterschreitung des vorgesehenen Mindestabstandes zu verhindern. Das beschriebene Vorgehen zeigt eine mit geringem Aufwand praktikable Möglichkeit, die aktuell in Deutschland geltenden Hygienerichtlinien einzuhalten. Mitarbeiter und Verantwortliche in den Kliniken befinden sich aktuell in einem veränderten Spannungsfeld zwischen Infektions- und Datenschutz. Der Infektionsschutz ist jedoch gerade für das dringend benötigte medizinische Personal in dieser Krise entscheidend. Zur Sicherung der medizinischen Versorgung in Deutschland sollten die allgemein geltenden Hygieneregeln auch vom medizinischen Personal eingehalten werden, wann immer es möglich ist. Für den flächendeckenden Einsatz von Telekommunikation sind dringend Vorgaben der Politik erforderlich, um die Teilnehmer regelmäßiger und wichtiger medizinischer Konferenzen, und damit Ihre Patienten, so gut wie möglich vor einer Infektion mit dem neuen Coronavirus zu schützen.

Interessenkonflikt

TK, AL, JG und OM geben an, dass kein Interessenkonflikt besteht. Für die Erstellung dieses Manuskriptes wurde keine finanzielle Unterstützung gewährt.

Literatur

[1] Kassenärztliche Bundesvereinigung. Anlage 31b zum Bundesmantelvertrag Ärzte (BMV-Ä). Im Internet: www.kbv.de/media/sp/Anlage_ 31_Telemedizinische_Leistungen.pdf; Stand: 27.03.2020

[2] Kassenärztliche Bundesvereinigung. Zertifizierte Videodienstanbieter. Im Internet: https://www.kbv.de/media/sp/Liste_zertifizierte_Videodienstanbieter.pdf; Stand: 28.03.2020

[3] Bundesärztekammer. Hinweise und Erläuterungen zu § 7 Absatz 4 MBO-Ä (Fernbehandlung). Im Internet: www.bundesaerztekammer.de/ fileadmin/user_upload/downloads/pdf-Ordner/Recht/2015-12-11_ Hinweise_und_Erlaeuterungen_zur_Fernbehandlung.pdf; Stand: 27.03.2020 